\title{
ANÁLISE DA PLANICIDADE DO GANHO DE AMPLIFICADORES HÍBRIDOS COM BOMBEAMENTO RESIDUAL
}

\author{
Márcia da Mota Jardim Martini ${ }^{1}$ \\ Fernando Almeida Diniz ${ }^{2}$ \\ Leonardo Paganotto ${ }^{3}$
}

\begin{abstract}
RESUMO
Neste trabalho são estudadas configurações de amplificadores híbridos constituídos por amplificador Raman em cascata com Erbium Doped Fiber Amplifier (EDFA) (Amplificador com fibra dopada com Érbio) com reciclagem de bombeamento residual Raman, a serem utilizados na expansão da capacidade de sistemas de comunicações óticas utilizando Wavelength Division Multiplexing (WDM) (Multiplexação por Divisão de Comprimento de Onda. Foi realizada), mediante simulação utilizando pacote comercial, análise do desempenho de diferentes configurações de amplificadores híbridos Raman+EDFA, em termos do ganho global e ondulação (ripple - variação do ganho dos canais de entrada). A flexibilidade do perfil espectral do amplificador Raman pode ser combinada com a alta capacidade de potência de saída do EDFA para obter amplificadores híbridos com banda larga. Este trabalho aplica a técnica de otimização de lasers de bombeamento de amplificadores Raman aplicada em amplificadores híbridos Raman+EDFA para aplicações em sistemas WDM. Os resultados preliminares demonstram que o amplificador híbrido Raman+EDFA com reciclagem de bombeamento residual Raman combinado com uma seleção apropriada de potências e comprimentos de onda dos lasers de bombeamento Raman, possibilita a obtenção de amplificadores híbridos banda larga com maior eficiência de conversão de potência, ganho alto e plano.
\end{abstract}

Palavras-chave: Amplificadores Raman. EDFA. Amplificadores híbridos. Bombeamento residual. WDM.

\section{GAIN RIPPLE ANALYSIS ON HYBRID AMPLIFIERS WITH RECYCLED PUMP}

\begin{abstract}
In this work different configurations of hybrid amplifiers are studied, made by a Raman amplifier followed by an EDFA. Such amplifiers can be used to increase the transmission capacity in optical communication systems. The performance of different hybrid EDFA+Raman amplifiers is obtained using commercial software. Their performance is analyzed in terms of the global gain and ripple. The flexibility of the Raman amplifier spectral

\footnotetext{
${ }^{1}$ Doutorado em Engenharia Elétrica e Informática Industrial pela Universidade Tecnológica Federal do Paraná, professora adjunta do Departamento de Física e Matemática do CEFET-MG. E-mail: marciamjmartini@yahoo.com.br

${ }^{2}$ Graduando em Engenharia Elétrica pelo CEFET-MG. E-mail: fernandoaldiniz@ gmail.com

${ }^{3}$ Graduando em Engenharia Elétrica pelo CEFET-MG. E-mail: lpaganotto@ hotmail.com
} 
profile combined with high capacity output power for EDFA provides broadband hybrid amplifiers. This paper uses the technique of optimizing lasers pumped Raman amplifiers applied in hybrid amplifiers for applications in Wavelength Division Multiplexing (WDM) systems. Preliminary results demonstrated that the Raman+EDFA hybrid amplifier under recycling residual Raman pump, allied with the proper selection of pump wavelengths and powers, enables the construction of broadband amplifiers with enhanced power conversion efficiency and high and flat gains.

Keywords: Raman amplifier. EDFA. Hybrid amplifiers. Pump recycling. WDM.

\section{INTRODUÇÃO}

O crescimento acelerado da demanda por tráfego de dados fez com que as comunicações por meio de sistemas óticos fossem cada vez mais exploradas. A tecnologia de transmissão de informação por fibra ótica é, atualmente, considerada a principal alternativa para aumentar a banda de transmissão, mantendo algumas características importantes como baixas atenuação e distorção do sinal, entre outros. Com isso, tem-se a necessidade de dispositivos construídos totalmente em fibras óticas, reduzindo custos na amplificação e regeneração da informação transmitida. O amplificador ótico tornou-se a ferramenta que permitiu a amplificação simultânea de um número grande de canais, ao contrário dos regeneradores eletrônicos que operavam canal por canal. A explosão da capacidade de enlaces de fibra ótica reside na multiplexação por divisão de comprimento de onda WDM.

A possibilidade de aplicações práticas tem direcionado esforços para o desenvolvimento de amplificadores óticos híbridos de baixo custo, confiáveis e eficientes que permitem o uso de uma ampla faixa espectral em sistemas WDM. Diversas configurações têm sido propostas na literatura de módulos discretos de amplificação com amplificadores híbridos usando reciclagem de bombeamento Raman residual. O conceito de compensação simultânea de perda e dispersão através da fibra compensadora de dispersão Dispersion compensation fiber (DCF) baseado em amplificação Raman tem atraído considerável atenção no desenvolvimento de amplificadores híbridos.

Lee et al. (2005a, 2005b) compararam experimentalmente o desempenho de amplificadores híbridos Raman + EDFA, no qual o ganho Raman é obtido na área de seção da fibra DCF. Em seu estudo, a potência do estágio Raman não aproveitada é reciclada como bombeamento para o estágio do EDFA. Foram estudadas várias configurações diferentes utilizando um único laser de bombeamento sobre DCFs. Eles observaram que reutilizando a potência de bombeamento Raman residual como bombeamento do EDFA, ocorre um 
acréscimo na eficiência do enlace ótico (os dois estágios de amplificação). O desempenho do amplificador foi medido em termos do ganho global, ondulação e figura de ruído Noise Figure (NF).

Estudo do desempenho de amplificadores híbridos tem sido realizado, inclusive experimentalmente, demonstrando que configurações utilizando reciclagem de bombeamento Raman fornecem amplificadores óticos com ganho alto e plano. No entanto, a implementação de diferentes configurações de amplificadores híbridos pode tornar-se onerosa e consequentemente inviável. Mais recentemente, a simulação numérica tem sido empregada para adicionar flexibilidade, como também para diminuir o tempo gasto para a análise de projetos dos amplificadores híbridos (LEE et al., 2005b, TIWARI; RAJAN; THYAGARAJAN, 2008).

Neste trabalho, os autores estendem a análise de amplificador híbrido Raman+EDFA, a partir de uma técnica que combina a equalização do espectro de ganho do EDFA por meio do ajuste de parâmetros do amplificador Raman. Como resultado, a variação do ganho global (ripple) é reduzida e o ganho médio global é melhorado comparado com os resultados apresentados na literatura.

O objetivo deste trabalho é estudar o desempenho de amplificadores híbridos Raman+EDFA, em onda contínua, utilizando bombeamento residual, para uso em sistemas WDM. Este trabalho consiste, principalmente, na análise das interações que ocorrem entre sinal-sinal, sinal-bombeamento e bombeamento-bombeamento. Este estudo poderá contribuir tanto para o conhecimento das vantagens e desvantagens de diferentes configurações quanto para mensurar a aplicabilidade de pacotes comerciais em novas proposições.

\section{DESENVOLVIMENTO}

\subsection{Metodologia}

A metodologia utilizada tenta ajustar o perfil do ganho do estágio Raman, de maneira que compense o ganho do segundo estágio de amplificação EDFA. A caracterização do amplificador híbrido Raman+EDFA proposto foi realizada através de simulações computacionais. Para efeito de simplificação, não são abordados efeitos de fase, de modulação cruzada e de mistura de quatro ondas. Resultados foram obtidos considerando configurações de bombeamento simples e duplo, como também múltiplos canais de entrada WDM (8 e 16 canais), considerando dois níveis de potência do sinal de entrada ( $-20 \mathrm{dBm}$ e 
$-10 \mathrm{dBm})$. A análise do desempenho do referido amplificador foi realizada em termos do ganho médio global e da variação do ganho global (ripple). A caracterização do ganho em cada estágio foi feita para melhor entendimento das interações que ocorrem entre sinal-sinal, sinal-bombeamento e bombeamento-bombeamento.

\subsection{Amplificador Híbrido Proposto}

A otimização de comprimentos de onda e de potências de múltiplos lasers de bombeamento é uma tarefa crítica que pode ser realizada durante o projeto do amplificador Raman. Um método analítico e preciso que permite o cálculo de ganho e de ripple foi proposto por Cani et al. (2009), com o objetivo de otimizar o projeto de amplificadores Raman. Este método equaliza o espectro do ganho do EDFA por meio do ajuste de parâmetros do amplificador Raman (potência e comprimento de onda de bombeamento). Baseado na técnica proposta por Martini et al. (2010a), o modelo analítico aproximado proposto por Cani et al. (2009) foi utilizado para determinar o perfil espectral do ganho Raman, evitando rotinas numéricas demoradas.

O método é dividido basicamente em duas etapas. Primeiramente, são gerados 100.000 perfis de ganho de amplificadores Raman, Cani et al. (2009), que são somados cada um ao perfil do ganho do EDFA de referência. Entre todos os perfis resultantes, são selecionados os 10 perfis que apresentaram ripple menor que $4 \mathrm{~dB}$. Na segunda etapa é usado o software comercial OptiSystem 7.0, que considera o amplificador híbrido completo, sendo as configurações selecionadas (potência e comprimentos de onda dos lasers de bombeamento Raman) e avaliadas como parâmetros de entrada, conforme apresentado na tabela 1. A configuração que obtém o menor variação do perfil do ganho global (ripple) dentre as configurações selecionadas, define os parâmetros da configuração final do amplificador híbrido resultante.

Tabela 1 - Parâmetros dos lasers de bombeamento usados nas simulações

\begin{tabular}{c|cc}
\hline $\begin{array}{c}\text { Número de } \\
\text { Lasers }\end{array}$ & $\begin{array}{c}\text { Comprimento de } \\
\text { onda }(\mathbf{n m})\end{array}$ & $\begin{array}{c}\text { Potência dos lasers } \\
(\mathbf{d B m})\end{array}$ \\
\hline $\mathbf{1}$ & 1425,0 & 23,64 \\
$\mathbf{2}$ & 1425,4 & 24,64 \\
& 1451,0 & 18,92 \\
\hline
\end{tabular}

Fonte: Adaptado de Martini (2010c) 
O amplificador híbrido proposto foi baseado na proposta apresentada por Lee et al. (2005b), conforme mostrado na figura 1. A configuração analisada é formada pelos estágios de amplificação Raman e EDFA, sendo que o primeiro estágio é constituído por 12,6 km de fibra DCF, área efetiva de $15,3 \mu \mathrm{m}^{2}$, coeficiente de atenuação de $0,5 \mathrm{~dB} / \mathrm{km}$ em $1550 \mathrm{~nm}$ e coeficiente de dispersão de -98 ps/(nm.km). O segundo estágio é constituído por $10 \mathrm{~m}$ de Fiber Doped Erbium (EDF) raio do núcleo de 2,2 $\mu \mathrm{m}$, área efetiva de 15,3 $\mu \mathrm{m}^{2}$ e concentração de Érbio de $1 \times 10^{25} \mathrm{~m}^{-3}$.

As simulações foram realizadas para amplificadores híbridos otimizados, considerando um e dois lasers de bombeamento Raman. Os isoladores óticos, acopladores WDM, combinadores de lasers e conectores de emenda neste documento são assumidos como componentes ideais.

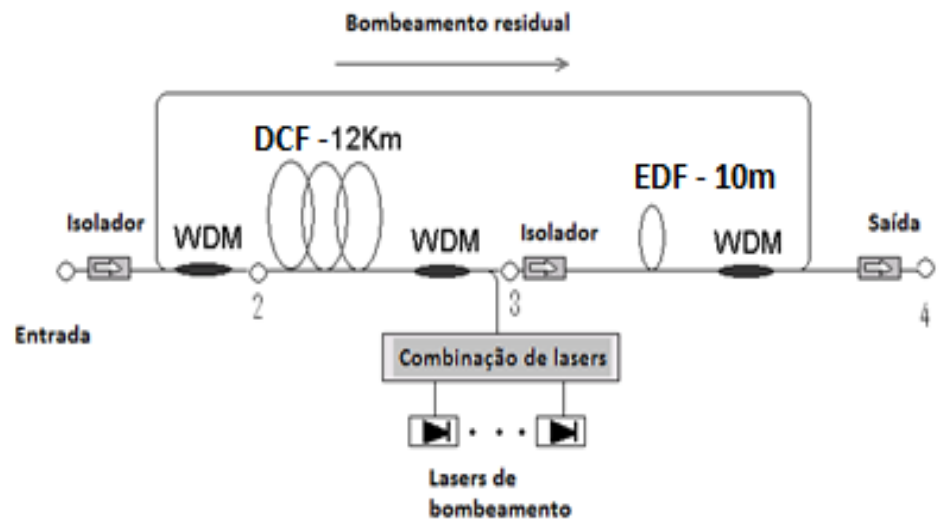

Figura 1 - Esquema do amplificador híbrido proposto Fonte: Adaptado de Lee et al. (2005b)

\subsection{Resultados e Discussão}

As simulações realizadas forneceram apenas alguns resultados preliminares que serão apresentados e discutidos nesta seção. Nas figuras 2 e 3 são apresentados resultados obtidos para análise do perfil de ganho de diferentes configurações de amplificador híbrido Raman+EDFA com bombeamento residual. As configurações foram modeladas no ambiente do software comercial OptiSystem ${ }^{\circledR}$ 7.0. Todos os parâmetros das simulações foram mapeados de acordo com as referências bibliográficas (experimentais) e o default do simulador utilizado.

A comparação do desempenho, em termos de ganho global médio e variação do ganho global de cada canal de entrada WDM, para diferentes configurações de amplificadores híbridos Raman+EDFA, foi realizada para verificar os benefícios e as desvantagens de cada 
configuração. Foi avaliada a possibilidade de fazer análise do ganho de amplificadores híbridos utilizando um software comercial, levando em consideração os problemas gerados pelas condições ideais dos componentes que integram o pacote computacional.

Os perfis do ganho dos estágios de amplificação Raman e EDFA, e do ganho global, apresentados nas figuras 2 e 3, são resultados obtidos considerando dois níveis de potência do sinal de entrada (-20 e $-10 \mathrm{dBm})$, com o objetivo de verificar a saturação do ganho do estágio EDFA e as mudanças ocorridas nos perfis de ganho, devido às interações sinal-sinal, bombeamento-bombeamento e sinal-bombeamento.

As primeiras simulações foram realizadas considerando bombeamento Raman simples, introduzindo, primeiramente, oito canais de entrada WDM, simultaneamente, sendo $-20 \mathrm{dBm}$ a potência de cada canal (figura 2a). Em seguida, foram introduzidos dezesseis canais WDM, nas mesmas condições (figura $2 b$ ).

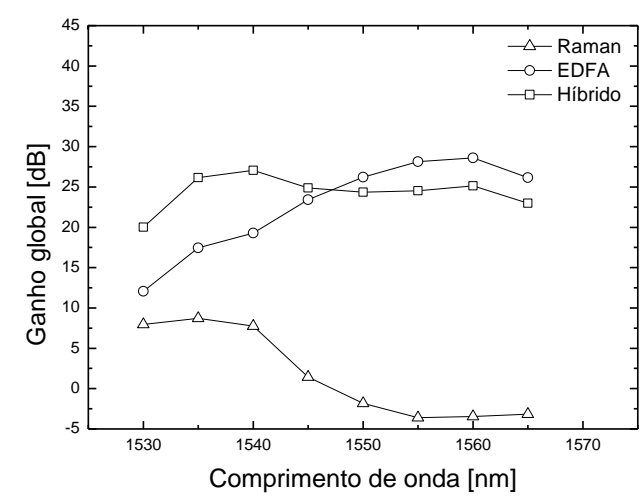

(a)

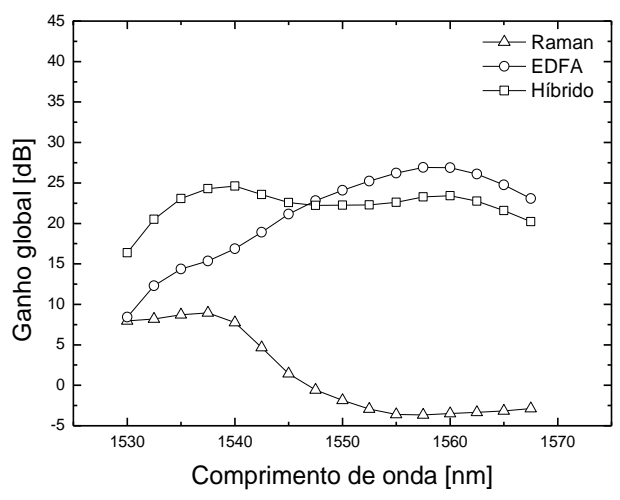

(b)

Figura 2 - Perfil do ganho global do amplificador híbrido proposto, considerando (a) oito canais e (b) 16 canais de entrada

Fonte: Adaptado de Diniz (2012)

Pode ser verificado que o aumento do número de canais de entrada resultou no valor médio do ganho global menor que $3 \mathrm{~dB}$, devido à saturação ocorrida no EDFA, principalmente nos canais com comprimento de onda menores que $1545 \mathrm{~nm}$. Entretanto, as mudanças ocorridas no perfil do ganho do estágio de amplificação Raman podem ser consideradas insignificantes.

Novas simulações foram realizadas, sendo considerado bombeamento Raman duplo, oito canais de entrada introduzidos simultaneamente, com potência de cada canal WDM de $-20 \mathrm{dBm}$. Os resultados obtidos são apresentados na figura 3a. Posteriormente, foram 
realizadas novas simulações considerando as mesmas condições, sendo a potência de cada canal de entrada WDM igual a $-10 \mathrm{dBm}$, conforme resultados apresentados na figura $3 \mathrm{~b}$.

$\mathrm{O}$ uso de dois lasers de bombeamento Raman aumentou o ganho global do amplificador híbrido EDFA+Raman de, aproximadamente, $5 \mathrm{~dB}$, enquanto que o ganho no estágio de amplificação Raman dobrou quando comparado com os resultados apresentados na figura 2a (bombeamento simples). Considerando a faixa de $1530 \mathrm{~nm}$ a $1545 \mathrm{~nm}$, foi verificado que a saturação ocorrida no EDFA, diminuiu, aproximadamente, $5 \mathrm{~dB}$, conforme pode ser observado na figura $3 \mathrm{a}$.

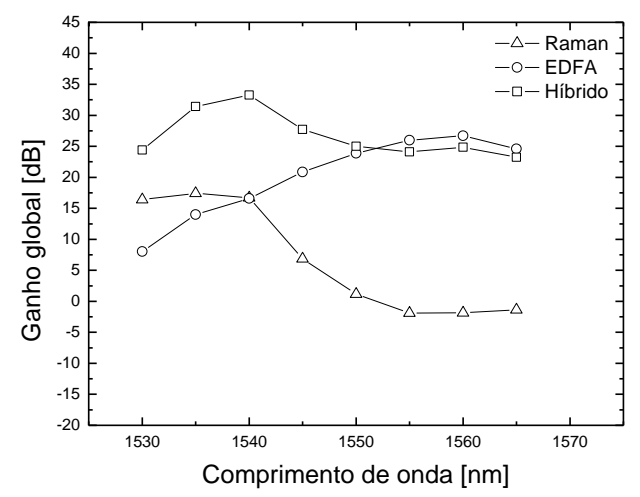

(a)

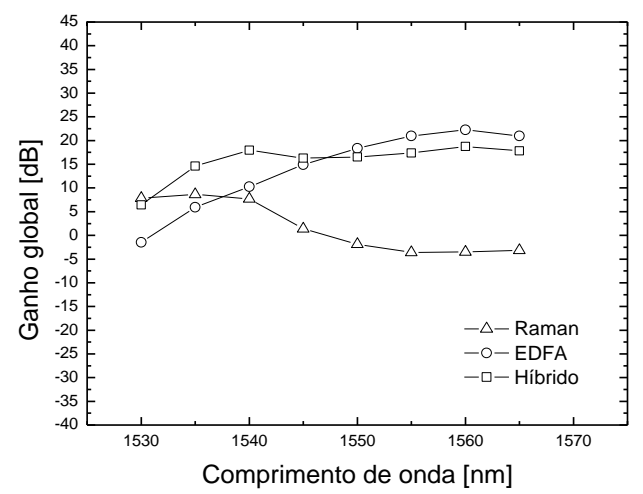

(b)

Figura 3 - Perfil do ganho global do amplificador híbrido proposto, considerando diferentes potências dos canais WDM. (a) $-20 \mathrm{dBm}$ e (b) $-10 \mathrm{dBm}$.

Fonte: Adaptado de Diniz (2012)

O desempenho do amplificador híbrido proposto pode ser considerado melhor, com dois lasers de bombeamento Raman e potência de $-20 \mathrm{dBm}$ em cada canal WDM, quando a análise é realizada em termos de ganho global (aumenta de aproximadamente $5 \mathrm{~dB}$ ). $\mathrm{O}$ resultado apresentado na figura 3.b mostra que o ganho global se torna menor, aproximadamente $10 \mathrm{~dB}$, considerando a potência de cada canal WDM de $-10 \mathrm{dBm}$, porém o ripple é de aproximadamente $2 \mathrm{~dB}$ na faixa de $1540 \mathrm{~nm}$ a $1565 \mathrm{~nm}$.

\section{CONCLUSÃO}

Os resultados preliminares obtidos demonstraram que a potência de entrada de cada canal de entrada WDM também é um parâmetro importante para a análise das interações sinal-sinal, sinal-bombeamento e bombeamento-bombeamento que ocorrem nos amplificadores híbridos. O aumento do número de canais de entrada faz com que o amplificador proposto tenha pior desempenho (ganho global $5 \mathrm{~dB}$ menor), pois os sinais com 
comprimentos de onda menores se comportam como bombeamento, transferindo energia para os sinais de comprimentos de onda maiores. O ganho dos canais de comprimentos de onda menores é reduzido, enquanto o ganho dos canais de comprimentos de onda maiores se tornam ainda maiores, o que, consequentemente, tende a aumentar o ripple. As transferências de energia (interações analisadas) que ocorrem com o aumento do número de bombeamentos otimizados proporcionam maior planicidade do ganho global, isto é, tais interações ajudam no desempenho do amplificador híbrido otimizado. Configurações que utilizam bombeamento Raman residual, combinado com escolha adequada de potência e comprimento de onda do laser de bombeamento Raman (otimização), permitem a obtenção de amplificadores óticos com banda larga e ganho alto.

\section{REFERÊNCIAS}

CANI, S. P. et al. An analytical approximated solution for the gain of broadband Raman amplifiers with multiple counter-pumps. Journal of Ligthwave Technology, v. 27, n. 7, p. 944-951, apr. 2009.

CASTELLANI, C. E. S. et al. Design methodology for multi-pumped discrete Raman amplifiers: case-study employing photonic crystal fibers. Optics Express, v. 17, n. 16, p. 14121-14131, aug. 2009.

DINIZ, F. A.; MARTINI, M. M. J. Gain ripple analysis on hybrid amplifiers with recycled pump under increased WDM channels. In: SIMPÓSIO BRASILEIRO DE MICRO-ONDAS E OPTOELETRÔNICA, 15., 2012, João Pessoa. Pôster... João Pessoa: IFPB, 2012.

DINIZ, F. A.; MARTINI, M. M. J.; PAGANOTTO, L. Análise da planicidade do ganho de amplificadores óticos com bombeamento Raman residual. In: REUNIÃO ANUAL DA SOCIADADE BRASILEIRA PARA O PROGRESSO DA CIÊNCIA, 65., 2013, Recife. Pôster... Recife: UFPE, 2013.

LEE, J. H. et al. Dispersion-compensating Raman/EDFA hybrid amplifier recycling residual Raman pump for efficiency enhancement. IEEE Photonics Technology Letters, v. 17, n. 1, p. 43-45, jan. 2005a.

LEE, J. H. et al. Performance comparison of various configurations of single-pump dispersion-compensating Raman/EDFA hybrid amplifiers. IEEE Photonics Technology Letters, v. 17, n. 4, p. 765-767, apr. 2005b. 
MARTINI, M. M. J. et al. Analysis of a multi-pump optimization in Raman+EDFA hybrid amplifiers with pump recycling for WDM systems. In: PHOTONICS EUROPE

INTERNATIONAL SYMPOSIUM, 2010, Brussels. Anais... Brussels: SPIE, 2010a, p. $12-$ 16.

MARTINI, M. M. J. Otimização de amplificadores híbridos RAMAN+EDFA utilizando reciclagem de bombeamento. 2010. 116f. Tese (Doutorado em Engenharia Elétrica) Programa de Pós-Graduação em Engenharia Elétrica e Informática Industrial, Universidade Tecnológica Federal do Paraná, Curitiba, 2010c.

MARTINI, M. M. J. Multi-pump optimization for Raman+EDFA hybrid amplifiers under pump residual recycling. In: INTERNATIONAL OPTOELECTRONICS CONFERENCE, 2009, Belém. Anais... Belém: IMOC, 2009, p. 117-121.

MARTINI, M. M. J. et al. Multi-pump optimization for Raman+EDFA hybrid amplifiers in WDM systems. Journal of Microwaves, Optoelectronics and Electromagnetic Applications, v. 9, n. 2, p. 100-112, dec. 2010b.

TIWARI, U.; RAJAN, K.; THYAGARAJAN, K. Multichannel gain and noise figure evaluation of Raman/EDFA hybrid amplifiers. Optics Communications, v. 6, n. 281, p. 1593-1597, mar. 2008.

TIWARI, U.; THYAGARAJAN, K.; SHENOY, M. R. Simulation and experimental characterization of Raman/EDFA hybrid amplifier with enhanced performance. Optics Communications, v. 8, n. 282, p. 1563-1566, apr. 2009.

Recebido em: 15/09/2013

Aprovado em: 03/10/2013 